TITLE:

\title{
Neural mechanisms underlying conscious and unconscious attentional shifts triggered by eye gaze.
}

\section{$\operatorname{AUTHOR}(\mathrm{S})$ :}

Sato, Wataru; Kochiyama, Takanori; Uono, Shota; Toichi, Motomi

\section{CITATION:}

Sato, Wataru ...[et al]. Neural mechanisms underlying conscious and unconscious attentional shifts triggered by eye gaze.. Neurolmage 2015, 124(Part A): 118-126

\section{ISSUE DATE:}

2015-09-04

URL:

http://hdl.handle.net/2433/200278

\section{RIGHT:}

(c) 2015. This manuscript version is made available under the CC-BY-NC-ND 4.0 license

http://creativecommons.org/licenses/by-nc-nd/4.0/; The full-text file will be made open to the public on 4 September 2016 in accordance with publisher's 'Terms and Conditions for Self-Archiving'.; この論文は出版社版でありません。引 用の際には出版社版をご確認ご利用ください。; This is not the published version. Please cite only the published version. 


\title{
Neural mechanisms underlying conscious and unconscious attentional shifts triggered by eye gaze
}

Wataru Sato ${ }^{1,2}$, Takanori Kochiyama ${ }^{1}$, Shota Uono ${ }^{2}$, and Motomi Toichi ${ }^{2,3}$

${ }^{1}$ The Hakubi Project, Primate Research Institute, Kyoto University, Aichi 484-8506, Japan. ${ }^{2}$ The Organization for Promoting Developmental Disorder Research, 40 Shogoin-Sannocho, Sakyo, Kyoto 606-8392, Japan. ${ }^{3}$ Faculty of Human Health Science, Graduate School of Medicine, Kyoto University, 53 Shogoin-Kawaharacho, Sakyo-ku, Kyoto 606-8507, Japan.

\begin{abstract}
Behavioral studies have shown that eye gaze triggers attentional shifts both with and without conscious awareness. However, the neural substrates of conscious and unconscious attentional shifts triggered by eye gaze remain unclear. To investigate this issue, we measured brain activity using event-related functional magnetic resonance imaging while participants observed averted or straight eye-gaze cues presented supraliminally or subliminally in the central visual field and then localized a subsequent target in the peripheral visual field. Reaction times for localizing the targets were shorter under both supraliminal and subliminal conditions when eye-gaze cues were directionally congruent with the target locations than when they were directionally neutral. Conjunction analyses revealed that a bilateral cortical network, including the middle temporal gyri, inferior parietal lobules, anterior cingulate cortices, and superior and middle frontal gyri, was activated more in response to averted eyes than to straight eyes under both supraliminal and subliminal conditions. Interaction analyses revealed that the right inferior parietal lobule was specifically active when participants viewed averted eyes relative to straight eyes under the supraliminal condition; the bilateral subcortical regions, including the superior colliculus and amygdala, and the middle
\end{abstract}


temporal and inferior frontal gyri in the right hemisphere were activated in response to averted versus straight eyes under the subliminal condition. These results suggest commonalities and differences in the neural mechanisms underlying conscious and unconscious attentional shifts triggered by eye gaze.

\section{Keywords}

amygdala; attentional shift; conscious awareness; eye gaze; fMRI; subliminal presentation

\section{Introduction}

The eyes of other individuals automatically trigger multiple psychological activities in observers (Kendon, 1967). For example, the perception of averted eyes may alert observers to critical information about the environment, such as dangerous animals, and allow rapid reactions to such stimuli. At the same time, averted eyes may signal the intention to share attentional focus with others and thereby create social coordination.

Consistent with these ideas, several behavioral studies have revealed that eye gaze can trigger attentional shifts under both conscious and unconscious conditions (Al-Janabi and Finkbeiner, 2012; Bailey et al., 2014; Sato et al., 2007; Sato et al., 2010; Xu et al., 2011). In these studies, researchers presented eye-gaze cues in the central visual field either supraliminally or subliminally using a cueing paradigm (cf. Posner, 1980). The results consistently showed that participants' reaction times (RTs) for processing targets were shorter when the targets were preceded by cues that were directionally congruent with the target locations than when they were preceded by directionally incongruent cues under both presentation conditions. This cueing effect was observed under both supraliminal and subliminal presentation conditions when the cues were not predictive of target locations (Bailey et al., 2014; Sato et al., 2007; Sato et al., 2010; Xu et al., 2011; however, see Al-Janabi and Finkbeiner, 2012) and when concurrent information load was high (Xu et al., 2011). 
These data suggest that attentional shifts are automatically triggered by eyes, following a common pattern with and without conscious awareness. At the same time, some of these studies found different patterns of attentional shifts across supraliminal and subliminal presentation conditions. Specifically, reduced cueing effects caused by eye cues were found under subliminal, but not supraliminal, conditions among individuals with autistic spectrum disorders compared with typically developing controls (Sato et al., 2010) and under supraliminal, but not subliminal, conditions in older adults relative to younger adults (Bailey et al., 2014). These dissociations suggest the involvement of different mechanisms in conscious and unconscious gaze-triggered attentional shifts. In summary, behavioral data suggest that both conscious and unconscious viewing of eyes can trigger attentional shifts, with certain commonalities and differences evident across conditions.

Neuroimaging studies have explored the neural mechanisms underlying attentional shifts triggered by consciously viewed eye gaze. A number of studies reported that the observation of averted eyes compared with straight eyes or other control stimuli, elicited more activation in several brain regions, including the posterior superior temporal sulcus/middle temporal gyrus (Calder et al., 2002; Engell and Haxby, 2007; Hoffman and Haxby, 2000; Hooker et a1., 2003; Pelphrey et al., 2003; Puce et al., 1998; Sato et al., 2008; Wicker et al., 1998), fusiform gyrus (Calder et al., 2002; George, et al., 2001; Pelphrey et al., 2003; Wicker et al., 1998), and inferior parietal lobule (Calder et al., 2002; Hoffman and Haxby, 2000; Pelphrey et al., 2003; Sato et al., 2008; Wicker et al., 1998), and middle/inferior frontal gyrus (Calder et al., 2002; Hooker et al., 2003; Wicker et al., 1998). Several studies investigated the brain activation in response to averted versus straight eyes in the framework of the cueing paradigm (Callejas et al., 2013; Cazzato et al., 2012; Engell et al., 2010; Greene et al., 2009; Hietanen et al., 2006; Kingstone et al., 2004; Sato et al., 2009; Tipper et al., 2008). Although foci, methodologies, and results were not identical across these studies and disagreements persist, several of these studies 
(Greene et al., 2009; Sato et al., 2009; Tipper et al., 2008) were consistent in reporting that the temporal, parietal, and frontal regions were involved in attentional shifts triggered by eye gaze. For example, Tipper et al. (2008) presented eye-gaze cues using a cueing paradigm and found activation in the distributed temporal, parietal, and frontal regions, including the superior temporal gyrus, inferior parietal lobule, and middle and inferior frontal gyri, during attentional shifts elicited by eye-gaze cues. Several neuroimaging studies also reported that these regions were active when attentional shifts were automatically triggered by non-social cues, such as peripheral sudden onset stimuli and centrally presented symbols (e.g., Rosen et al., 1999; for a review, see Grosbras et al., 2005). Several studies reported that similar brain regions were activated for attentional shifts, regardless of whether they were triggered by eye gaze or non-social cues (Greene et al., 2009; Sato et al., 2009; Tipper et al., 2008; however, see Hietanen et al., 2006). Based on this evidence, it has been proposed that these regions constitute the attentional neural network (Corbetta and Shulman, 2002; Grosbras et al., 2005). Taken together, these findings suggest that conscious attentional shifts induced by gaze are implemented by the activation of the temporo-parieto-frontal cortical attentional network.

However, questions about whether the neural mechanisms underpinning attentional shifts triggered by consciously and unconsciously perceived gaze could be common or different remain unanswered. No study has examined this issue. However, the aforementioned behavioral data showing commonalities across conscious and unconscious gaze-triggered attentional shifts suggest commonalities in the neural substrates. Several neuroimaging studies also reported common patterns in neural activation regarding the conscious and unconscious processing of facial stimuli (Jiang and He, 2006; Morris et al., 2007; Prochnow et al., 2013). Based on these data, we hypothesized that the temporo-parieto-frontal attentional network would be involved in both conscious and unconscious gaze-triggered 
attentional shifts.

Additionally, based on the behavioral data, we expected to find several differences between conscious and unconscious gaze-triggered attentional shifts. Neuroimaging studies have provided indirect evidence related to this issue, reporting that the amygdala was involved in the processing of subliminally presented facial stimuli (e.g., Morris et al., 1998; Whalen et al., 1998) and specifically activated in response to subliminally presented fearful versus neutral eyes (Whalen et al., 2004). A neuroimaging study reported that the activity of the amygdala of a patient with damage to the entrance of the cortical visual areas changed depending on the direction of unseen eyes (Burra et al., 2013). An intracranial electroencephalography study reported that amygdala activation in response to eyes was rapid, indicating that it can occur prior to or simultaneously with the conscious awareness of faces (Sato et al., 2011, 2013). Several neuroimaging studies also found that emotional facial expressions, which are integratively processed with gaze direction (e.g., Sato et al., 2004), were unconsciously processed through the subcortical visual pathway to the amygdala, which includes the superior colliculus and pulvinar (e.g., Morris et al., 2001; for a review, see Tamietto and de Gelder, 2010). Further, the visual pathways involved in processing conscious and unconscious emotional facial expressions differed (e.g., Vuilleumier et al., 2002; for a review, see Vuilleumier and Pourtois, 2007). These data suggest the involvement of subcortical structures in the unconscious processing of eyes. Although direct evidence is lacking, based on these studies together with behavioral data suggesting specific mechanisms for unconscious gaze-triggered attentional shifts, we hypothesized that subcortical structures would be specifically related to unconscious attentional shifts triggered by gaze.

To test these hypotheses, we measured brain activity using rapid event-related functional magnetic resonance imaging (fMRI) while participants observed averted and straight eyes presented supraliminally or subliminally in the central visual field 
and then localized a subsequent target in the peripheral visual field. We performed cognitive conjunction analysis with interaction masking (Price and Friston, 1997) to identify commonalities in brain activity in response to averted versus straight eyes across presentation conditions. We also examined differences in brain activity by analyzing interactions between gaze direction and presentation condition.

\section{Methods}

Participants

Twenty-seven volunteers ( 3 women and 24 men; mean $\pm S D$ age, $25.0 \pm 4.6$ years) participated in the experiment. All participants were right-handed, as assessed by the Edinburgh Handedness Inventory (Oldfield, 1971), and had normal or corrected-to-normal visual acuity. After the experimental procedures were fully explained, all participants provided informed consent regarding their participation. This study was approved by the local institutional ethics committee.

Experimental design

The fMRI analysis relied on a within-subject two-factorial design, including presentation condition (subliminal or supraliminal) and directional condition (averted or straight). Cue-target congruence (i.e., congruence between the cue's direction and the target's location: congruent, neutral, incongruent) was also included in the behavioral data analysis.

\section{Stimuli}

The eye-gaze stimuli were almost identical to those used in a previous behavioral study (Uono et al., 2009) (Fig. 1). We selected the cue stimuli from a standard set (Ekman and Friesen, 1976). Photographs of two models (one female and one male) showing a neutral facial expression were selected and manipulated. To manipulate gaze direction, the irises and pupils of the eyes were extracted from the original photographs and inserted at the right or left side of the eyeball using Adobe Photoshop 5.0. We cropped the photographs in an elliptical shape, $2.7^{\circ}$ wide and 
$3.8^{\circ}$ high, to exclude hair and background.

A mosaic image was created from a neutral facial expression by dividing the photos into a $50 \times 40$ grid and randomly reordering the pieces, rendering the resulting photograph unrecognizable as a face. The letter " $\mathrm{T}$ " $\left(0.6^{\circ}\right.$ wide $\left.\times 0.6^{\circ} \mathrm{high}\right)$, presented $5.7^{\circ}$ to the left or right of the center of the screen, was used as a target stimulus.

\section{Presentation apparatus}

The events were controlled by Presentation Software version 10.0 (Neurobehavioral Systems, Albany, CA, USA) implemented on a computer using Microsoft Windows. The stimuli were projected from a liquid crystal projector (DLA-G150CL; Victor Electronics, Brussels, Belgium) at a refresh rate of $75 \mathrm{~Hz}$ to a mirror positioned in a scanner in front of the participants.
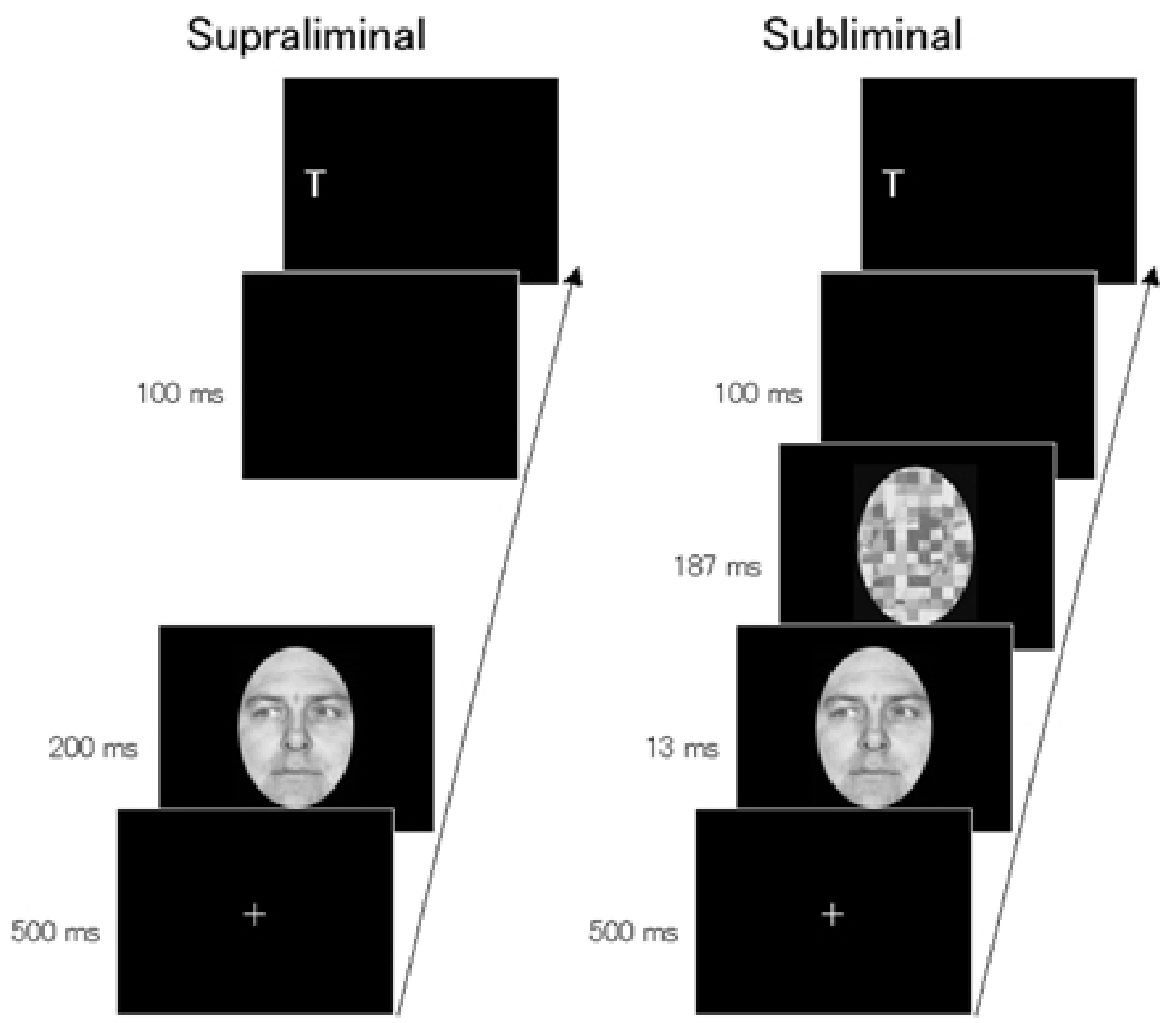

Figure 1. Illustrations of stimulus presentations. 


\section{Procedure}

The participants completed a total of 240 trials presented in two runs of 120 trials lasting $427.5 \mathrm{~s}$. Each run corresponded to one of the presentation conditions (supraliminal or subliminal), and the order of the conditions was counterbalanced across participants. We tested the presentation conditions in separate runs to prevent the participants from suspecting that eye-gaze cues were being presented under the subliminal condition (i.e., to make the participants believe that they were engaged in two different tasks), following several previous behavioral studies (Bailey et al., 2014; Sato et al., 2007, 2010). Each run consisted of an equal number of trials representing each of the gaze-direction conditions (i.e., 40 trials each of averted-left, averted-right, and straight eye-gaze conditions) and cue-target congruence conditions (i.e., 40 trials each of congruent, neutral, and incongruent conditions; 20 congruent and 20 incongruent trials for each averted-left and averted-right condition and 40 neutral trials for the straight condition), and the order of these conditions was randomized within each run. A break of approximately 1 min was inserted after the first run. Ten practice trials preceded the experimental trials.

A fixation point (i.e., a small black “+”) was presented for $500 \mathrm{~ms}$ at the center of the screen at the beginning of each trial (Fig. 1). The gaze cue was then presented at the same location. Under the supraliminal condition, the gaze cue was presented for $200 \mathrm{~ms}$, and no masking followed. Under the subliminal condition, the gaze cue was presented for $13 \mathrm{~ms}$ and was followed by the presentation of the mask in the same location for $187 \mathrm{~ms}$. Then, a target was presented in either the left or right visual field $\left(5.0^{\circ}\right.$ from the center) $100 \mathrm{~ms}$ after the gaze cue (under the supraliminal condition) or mask (under the subliminal condition) disappeared; the target remained until a response was made or $1,700 \mathrm{~ms}$ elapsed. As in previous studies (Friesen and Kingstone, 1998; Sato et al., 2007), participants were instructed to indicate as quickly as possible whether targets appeared on the left or right side of 
the monitor by pressing a key on the switch box using their left or right index finger to indicate left- or right-side locations, respectively. Participants were told that the stimuli preceding the targets were not predictive.

Our experimental design was based on a rapid event-related paradigm in which the efficiency of the design depended on the temporal pattern of stimulus or trial presentations (Dale, 1999; Friston et al., 1999). We maximized the efficiency with which we detected differential activation for averted and straight eyes while also maximizing the efficiency with which we estimated the evoked response under each condition. To ensure the latter, a null event was included, which occurred at a probability of $25 \%$ of all events. Accordingly, inter-trial intervals varied among $2,500,5,000,7,500,10,000$, and $12,500 \mathrm{~ms}$. The efficiency of the contrast estimates was evaluated using the inverted trace of the covariance matrix of the contrasts (Dale, 1999; Friston et al., 1999; cf. Morita et al., 2008). Of the 200,000 randomly generated design matrices, we selected the two most efficient in each run under the supraliminal and subliminal conditions.

Because we tested the effect of presentation condition in separate runs, we also evaluated the efficiency of this particular design. We compared three different experimental designs: (1) the current design (each presentation condition was tested separately in each of two runs), (2) mixed (both presentation conditions were tested together in two runs of blocked trials), and (3) randomized (both presentation conditions were tested together in each run, occurring as inter-mixed randomized trials). Efficiency was evaluated using the same method as the aforementioned simulation (i.e., 200,000 random generations of design matrices and an evaluation of the inverted trace of the covariance matrix of the contrasts). The contrasts of interest consisted of the differential activation for averted versus straight eyes within and across presentation conditions. To take into account the effects of temporal filtering (Friston et al., 2000), we also computed the modified efficiency by applying a high-pass filter of 128-s cut-off and a first-order autoregressive model 
(autoregressive coefficient: 0.3 ). The results of the simulation showed that the current design had the maximum efficiency (current $>$ mixed $>$ randomized) for both types of measures.

To ensure that the subliminal cue stimuli were presented without the participants' conscious awareness, we assessed the subjective thresholds of participants after MRI image acquisition with a procedure similar to those used in previous behavioral studies (Sato et al., 2007, 2010). The participants completed a total of 30 trials, 24 of which were similar to the trials under the subliminal condition during image acquisition, except that the gaze cues were presented for 13 , 27,40 , and $53 \mathrm{~ms}$ in each of six trials. We also included six trials with no gaze cue (i.e., mask image only) as the baseline condition (to consider cases of false-positive responses). The order of trials was randomized. Participants were asked, "Did you see the gaze? If so, report the direction of the gaze." Participants responded either "Yes" or "No;" in the case of a "Yes" response, they reported the gaze direction that they had seen.

\section{MRI acquisition}

Image scanning was performed on a 3-T scanning system (MAGNETOM Trio A, Tim System; Siemens, Malvern, PA, USA) using a 12-channel head coil. A forehead pad was used to stabilize the head position. The functional images consisted of 40 consecutive slices parallel to the anterior-posterior commissure plane, covering the whole brain. A T2*-weighted gradient-echo echo-planar imaging sequence was used with the following parameters: repetition time $(\mathrm{TR})=2,500$; echo time $(\mathrm{TE})=30$ ms; flip angle $=90^{\circ}$; matrix size $=64 \times 64$; voxel size $=3 \times 3 \times 4 \mathrm{~mm}$. After the acquisition of functional images, a T1-weighted high-resolution anatomical image was obtained using a magnetization-prepared rapid-acquisition gradient-echo sequence $\left(\mathrm{TR}=2,250 \mathrm{~ms} ; \mathrm{TE}=3.06 \mathrm{~ms} ;\right.$ flip angle $=9^{\circ} ;$ field of view $=256 \times 256$ $\mathrm{mm}$; voxel size $=1 \times 1 \times 1 \mathrm{~mm})$. 
The median correct RT under each condition was calculated for each participant. To satisfy normality assumptions for the subsequent analyses, these data were subjected to log transformation. Then, the log-transformed RTs were analyzed using a 3 (cue-target congruence: congruent, neutral, or incongruent) $\times 2$ (presentation condition: subliminal or supraliminal) repeated-measures analysis of variance (ANOVA). For significant interactions, the simple effects of cue-target congruence were analyzed, based on our interests, using one-tailed $t$-statistics. Preliminary analyses were conducted for error percentages. The error rates were small $(<5 \%)$, and we found no evidence of a speed-accuracy trade-off. Hence, we report only the RT results.

In terms of threshold assessment, we conducted a series of paired $t$-tests comparing the "Yes (seen)" responses under the no-gaze condition with 13-, 27-, 40-, or 53-ms presentation times. We also examined whether correct responses under the 13-, 27-, 40-, or 53-ms presentation conditions exceeded the level of chance (random selection; i.e., 25\%) using one-sample $t$-tests.

Results of all tests were considered statistically significant at $p<.05$. Image analysis

Image and statistical analyses were performed using the statistical parametric mapping package SPM8 (http://www.fil.ion.ucl.ac.uk/spm), implemented in MATLAB R2009a (MathWorks Inc., Natick, MA, USA). Functional images of each run were realigned using the first scan as a reference to correct for head movements. Data from all 27 participants required only small motion correction $(<2 \mathrm{~mm})$. Then, T1 anatomical images were coregistered to the first scan of the functional images. Following this, the coregistered $\mathrm{T} 1$ anatomical image was normalized to the Montreal Neurological Institute space using the unified segmentation-spatial normalization approach (Ashburner and Friston, 2005). The parameters from this normalization process were then applied to each of the functional images. Finally, these spatially normalized functional images were resampled to a voxel size of $2 \times$ 
$2 \times 2$ and smoothed with an isotopic Gaussian kernel of $8-\mathrm{mm}$ full-width at half-maximum to compensate for anatomical variability among participants.

We used random-effects analyses to identify significantly activated voxels at the population level (Holmes and Friston, 1998). First, we performed a single-subject analysis (Friston et al., 1995). The presentation of each condition was embedded in a series of delta functions. The task-related regressor was modeled by convolving it with a canonical hemodynamic response function. We used a high-pass filter composed of a discrete cosine basis function with a cutoff period of 128 to eliminate the artifactual low-frequency trend. To correct the global fluctuation related to motion artifacts, global scaling was conducted. Serial autocorrelation, assuming a first-order autoregressive model, was estimated from the pooled active voxels with a restricted maximum likelihood procedure and was used to whiten the data and the design matrix (Friston et al. 2002).

Initially, the contrast between averted and straight eyes was tested for each presentation condition. Voxels were identified as significantly activated if they reached a height threshold of $p<.01$ (uncorrected), with an extent threshold of 30 voxels $\left(240 \mathrm{~mm}^{3}\right)$. These analyses were conducted as exploratory analyses for the following statistical tests of commonalities and differences.

Next, to test for commonalities in brain activity in response to averted versus straight eyes across presentation conditions, we performed a conjunction analysis using interaction masking (Price and Friston, 1997) as in a previous study (Sato et al., 2009). For this analysis, we conducted a main-effect analysis of directional condition (averted versus straight) using $T$-statistics. To search for brain areas that showed similar activity across presentation conditions (supraliminal and subliminal), the main effect was exclusively masked by the $F$-tests of interactions. Voxels showing significant interactions between effects at a threshold of $p<.05$ (uncorrected) were eliminated from the statistical parametric map of the main effect. For the main effect contrast, voxels were identified as significantly activated if they 
reached the height threshold of $p<.01$ (uncorrected) with the extent threshold of 100 contiguous voxels $\left(800 \mathrm{~mm}^{3}\right)$, which roughly corresponded to $p<.05$ (corrected) determined by Monte Carlo simulations (e.g., 77 voxels; Ramasubbu et al., 2014), to produce the best balance between Type I and Type II errors (Lieberman and Cunningham, 2009).

Finally, to test for differences in brain activity for averted versus straight eyes across presentation conditions, interactions between direction and presentation condition were analyzed. We analyzed the specific instances in which higher levels of activity were more strongly associated with one presentation condition than with another. Thresholds were identical to those used in the aforementioned commonality analysis.

The brain structures were anatomically labeled using Talairach Client (http://www.talairach.org/) (Lancaster et al., 2007) and the Automated Anatomical Labelling atlas (Tzourio-Mazoyer et al., 2002) provided by the MRIcron software (http://www.mccauslandcenter.sc.edu/mricro/mricron/). We also identified certain brain regions based on visual inspection of anatomical MRI with reference to a standard atlas (Mai et al., 1997).

\section{Results}

\section{Threshold assessment}

The mean $\pm S E$ percentages of "Yes $($ seen)" responses were $5.6 \pm 3.0,6.2 \pm 2.4$, $4.9 \pm 2.3,18.5 \pm 4.1$, and $40.7 \pm 7.0 \%$ under the no-gaze, 13-, 27-, 40-, and 53-ms presentation conditions, respectively. We found significant differences between the percentage of "Yes (seen)" responses under the no-gaze, 40-, and 53-ms presentation conditions $(t(26)>2.90, p<.01)$ but not under the no-gaze, 13-, and 27-ms conditions $(t(26)<0.29, p>.10)$.

The mean $\pm S E$ percentages of correct responses were $4.3 \pm 1.7,2.5 \pm 1.5,12.3$ \pm 3.2 , and $30.9 \pm 6.0 \%$ under the 13-, 27-, 40-, and 53-ms presentation conditions, 
respectively. The percentages of correct responses were significantly lower than chance under the 13-, 27-, and 40-ms presentation conditions $(t(26)>4.01, p<.001)$ but not under the 53-ms presentation condition $(t(26)=0.98, p>.10)$.

The results confirmed that the subliminal cue stimuli under the current (i.e., 13-ms) condition were presented without eliciting participants' conscious awareness.

$R T$

For the correct RT (Table 1) after log-transformation, the ANOVA revealed a significant main effect of cue-target congruence and a significant interaction of cue-target congruence $\times$ presentation condition $(F(2,52)=21.62$ and 10.89 , respectively, all $p<.001)$. The main effect of presentation condition was not significant $(F(1,26)=2.25, p<.1)$.

Follow-up analyses of the interaction indicated that the RTs for congruent cues were significantly shorter than those for neutral cues under both supraliminal and subliminal presentation conditions $(t(104)=4.51$ and $1.83, p<.001$ and .05 , respectively). The RTs were also significantly shorter for congruent than for incongruent cues and for neutral than for incongruent cues under the supraliminal condition $(t(104)=7.56$ and $3.05, p<.001$ and .005 , respectively $)$, but not under the subliminal condition $(t(104)<1.35, p>.10)$.

Neural activity under each presentation condition

The contrast between averted and straight eyes was tested for each presentation condition (Table 2). Under each presentation condition, significant activities were detected in the bilateral parietal and right frontal regions, which partially overlapped between presentation conditions. Activities in the bilateral temporal

Table 1. Mean (SE) RTs under each cue-target relationship condition.

\begin{tabular}{lllllll}
\hline Presentation & \multicolumn{2}{c}{ Congruent } & Neutral & \multicolumn{3}{c}{ Inconguent } \\
\hline Supraliminal & 306.5 & $(26.1)$ & 314.6 & $(27.2)$ & 319.6 & $(27.4)$ \\
Subliminal & 308.5 & $(28.0)$ & 311.1 & $(27.9)$ & 308.9 & $(25.7)$ \\
\hline
\end{tabular}




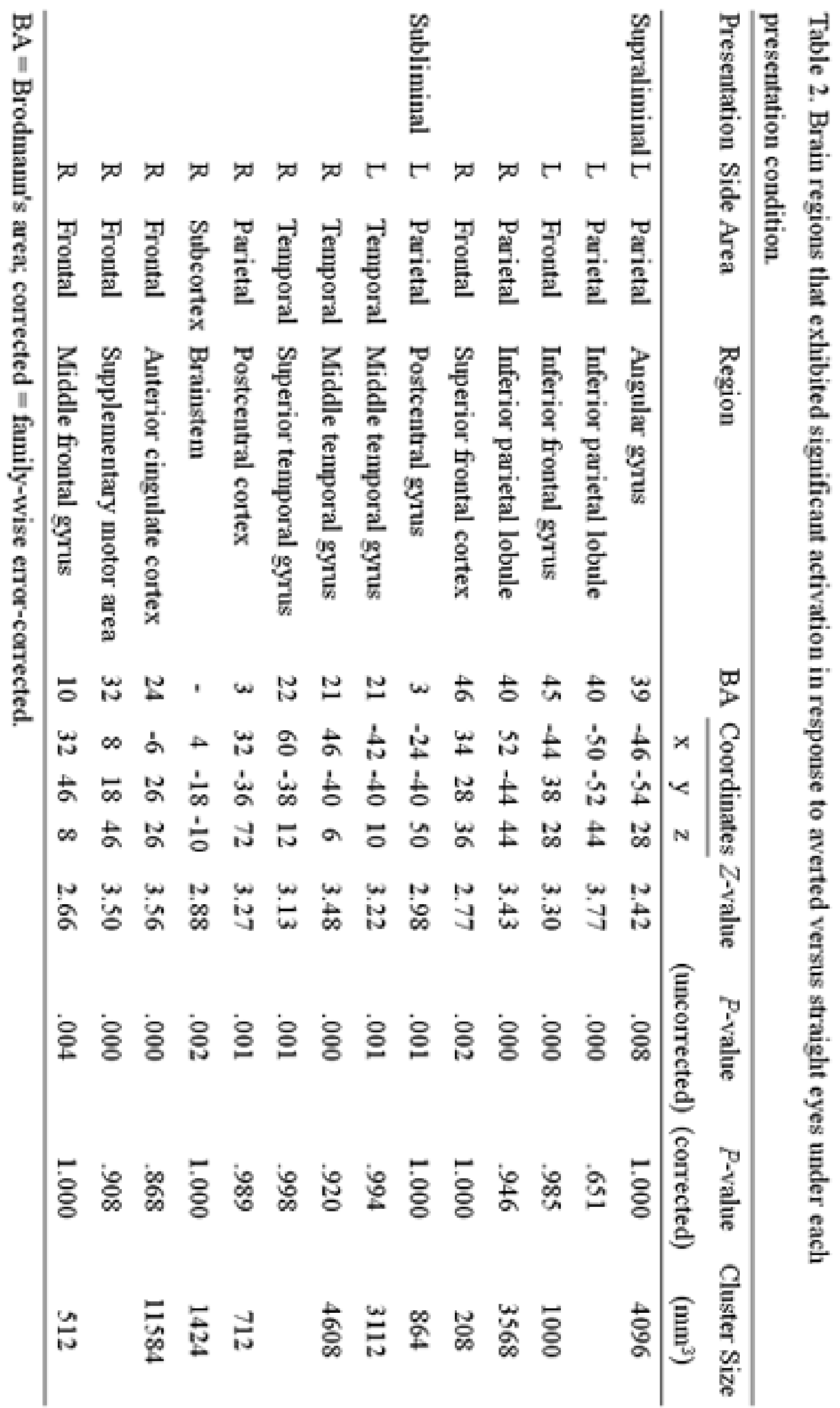


regions and anterior cingulate cortices were found under the subliminal presentation condition, and this was also the case under the supraliminal condition when a more liberal height threshold was used $(p<.05$, uncorrected). Significant activity was found in the brainstem under only the subliminal condition; the cluster included several other adjacent regions, including the amygdala, with a more liberal height threshold $(p<.05$, uncorrected).

Commonalities in neural activity

The conjunction analysis using interaction masking (Price and Friston, 1997) revealed that the averted versus straight eyes significantly activated temporal, parietal, and frontal cortical regions commonly under the subliminal and supraliminal presentation conditions. These regions included the middle temporal gyrus (covering the superior temporal sulcus) in the left hemisphere, the inferior parietal lobules and anterior cingulate cortices in both hemispheres, and the superior and middle frontal gyri in the right hemisphere (Table 3; Fig. 2).
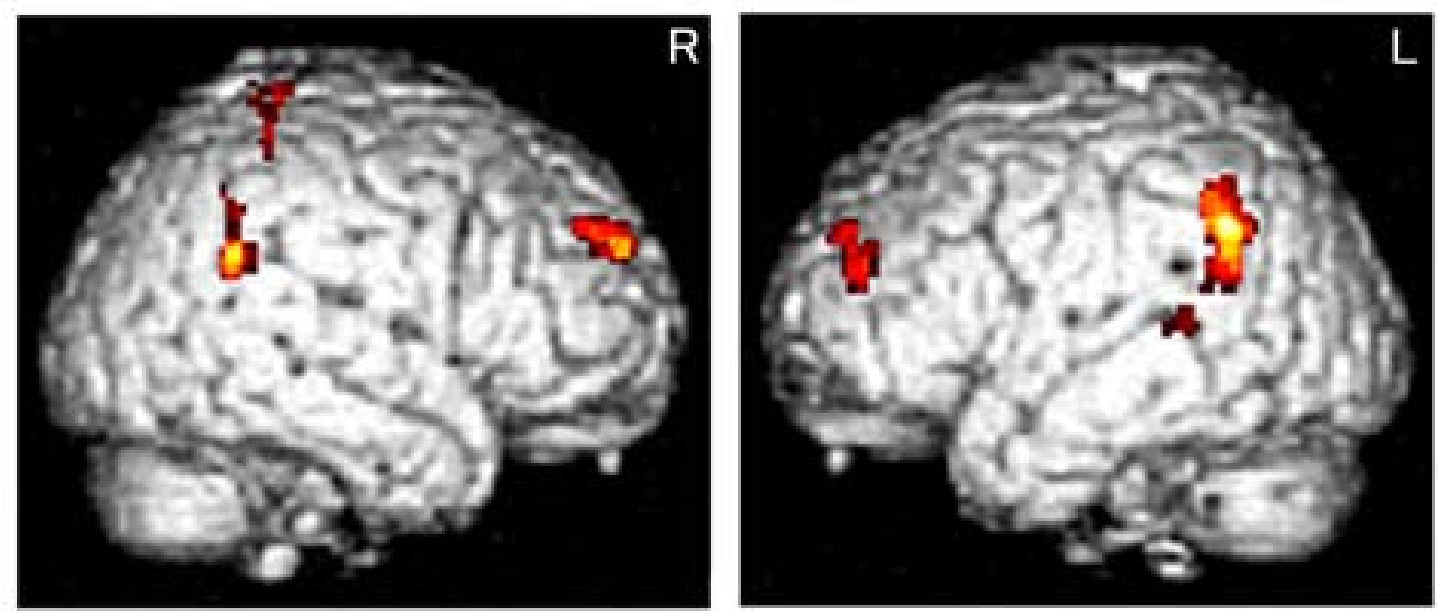

Figure 2. Statistical parametric maps indicating brain regions that were significantly activated in response to averted eyes compared with straight eyes under both supraliminal and subliminal presentation conditions. Areas of activation are rendered on spatially normalized brains. L, left hemisphere; R, right hemisphere. 


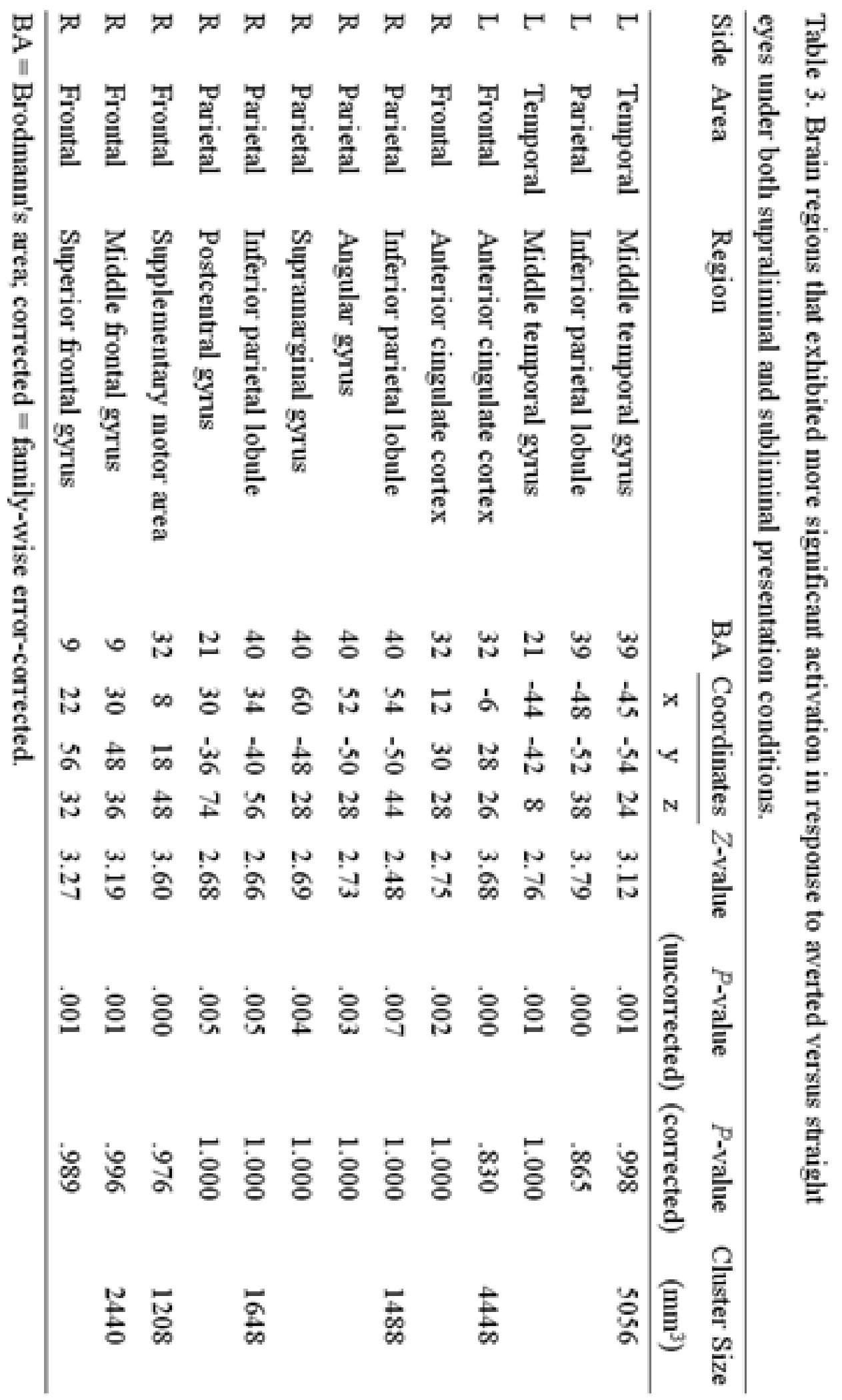


NeuroImage 18

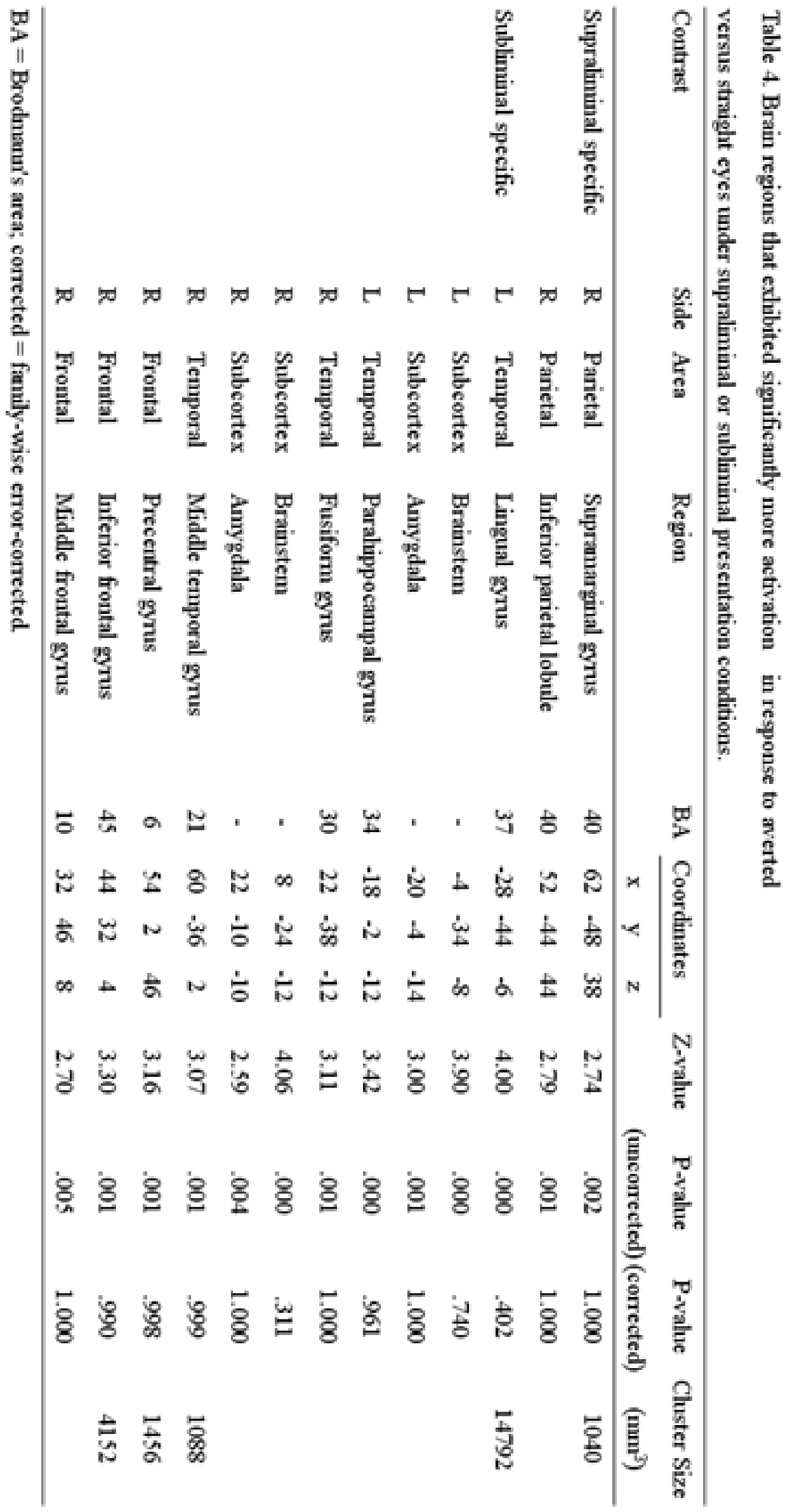




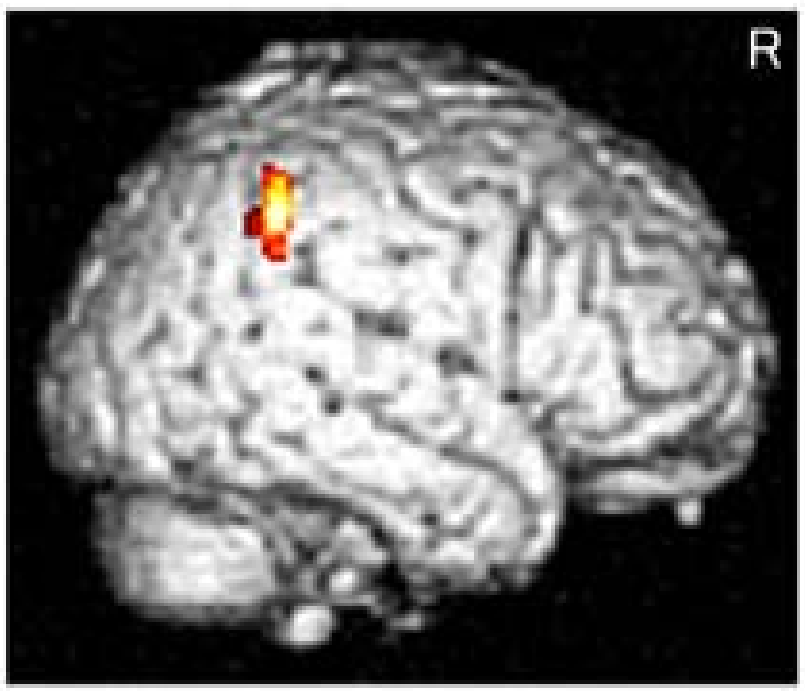

Figure 3. Statistical parametric maps indicating brain regions that were significantly more activated in response to averted versus straight eyes under the supraliminal than under the subliminal presentation condition. Areas of activation are rendered on spatially normalized brains. R, right hemisphere.
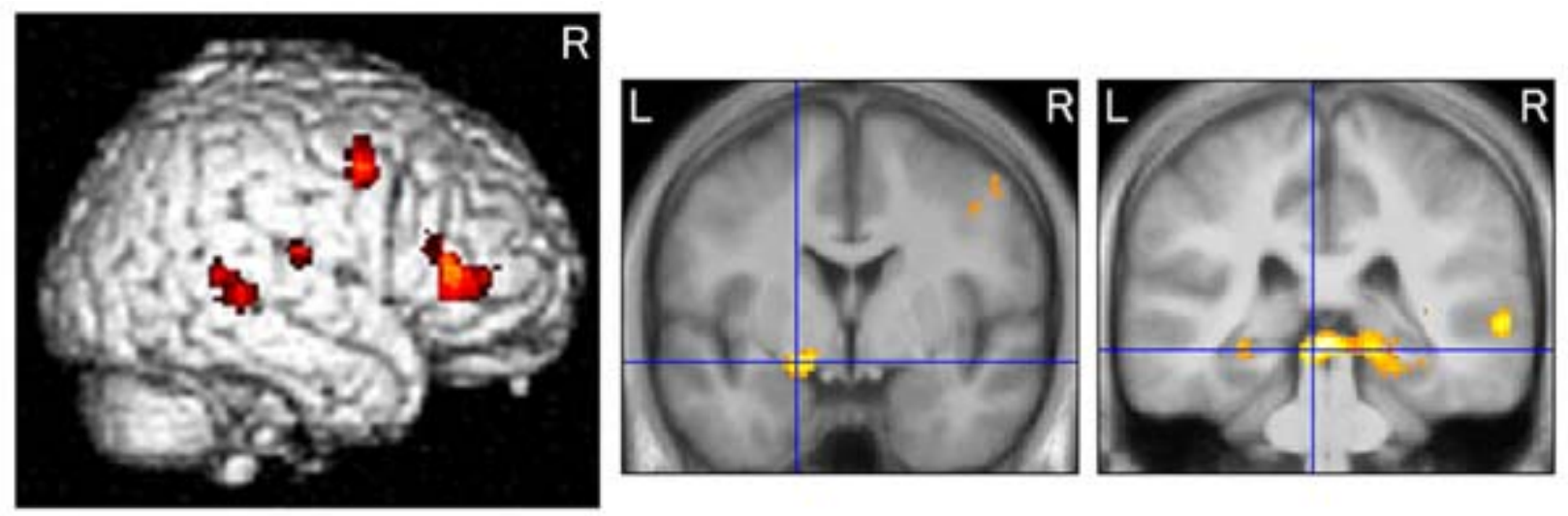

Figure 4. Statistical parametric maps indicating brain regions that were significantly more activated under the subliminal than under the supraliminal presentation condition in response to averted versus straight eyes. Areas of activation are rendered on spatially normalized brains (left) and overlaid on the mean normalized structural MRI from all participants in this study at the locations of the amygdala (middle) and superior colliculus (right). L, left hemisphere; R, right hemisphere. 


\section{Differences in neural activity}

Interaction analysis revealed significantly higher activity in the right inferior parietal lobule in response to averted versus straight eyes under the supraliminal condition than under the subliminal condition (Table 4, Fig. 3).

Significant activations for averted versus straight eyes under the subliminal condition relative to the supraliminal condition were found in broad subcortical regions, including the superior colliculus (the top of the midbrain; Schneider and Kastner, 2005) and amygdala (Table 4, Fig. 4). The contrast also revealed significant activations in the temporal and frontal cortices in the right hemisphere, including the middle temporal gyrus (covering the superior temporal sulcus), precentral gyrus, and middle and inferior frontal gyri (Fig. 4).

\section{Discussion}

Our behavioral results showed that congruent cues induced shorter RTs than did neutral cues under both supraliminal and subliminal presentation conditions. These results are consistent with those of previous studies (e.g., Bailey et al., 2014) and indicate that attentional shifts can be triggered both by supraliminally and subliminally presented eyes.

More importantly, our conjunction analyses of fMRI data revealed that widespread cortical regions were more activated in response to averted than to straight eyes under both the supraliminal and subliminal presentation conditions. These regions included the middle temporal gyrus, inferior parietal lobule, anterior cingulate cortex, and superior and middle frontal gyri. The activation of these regions is consistent with the results of several previous neuroimaging studies that investigated brain activation associated with the observation of averted eyes (e.g., Pelphrey et al., 2003) and attentional shifts triggered by averted eyes (e.g., Greene et al., 2009). However, these previous studies tested only the brain activities associated with the processing of consciously perceived eyes. Our results extend 
these previous findings and indicate that the temporo-parieto-frontal attentional network is involved in both conscious and unconscious gaze-triggered attentional shifts.

Furthermore, our interaction analyses revealed that several brain regions were more involved in attentional shifts triggered by subliminal than supraliminal eye-gaze cues. These regions included broad bilateral subcortical regions, including the superior colliculus and amygdala, as well as the middle temporal gyrus, precentral gyrus, and middle and inferior frontal gyri in the right hemisphere. The activation of the amygdala is consistent with previous neuroimaging evidence showing that the amygdala was active in response to subliminally presented faces (Morris et al., 1998; Whalen et al., 1998) and eyes (Burra et al., 2013; Whalen et al., 2004). The co-activation of the superior colliculus and amygdala corroborates neuroimaging data showing that these two regions were activated and functionally coupled during processing of unseen facial stimuli (Morris et al., 2001; cf. Pessoa and Adolphs, 2010; Tamietto and de Gelder, 2010). The involvement of several cortical regions is consistent with numerous neuroimaging studies reporting that both subcortical and cortical regions were involved in the unconscious processing of facial stimuli (Duan et al., 2010; Jiang and He, 2006; Morris et al., 2007; Phillips et al., 2004; Prochnow et al., 2013; Yang et al., 2012). Note, however, that our findings do not indicate that these regions are involved in only the unconscious processing of eye gaze. Several previous neuroimaging and neuropsychological studies reported that these brain regions, including the amygdala (Akiyama et al., 2007; Okada et al., 2008; Sato et al., 2009), superior/middle temporal gyrus (Akiyama et al., 2006; Greene et al., 2009; Sato et al., 2009; Tipper et al., 2008), precentral gyrus (Greene et al., 2009; Tipper et al., 2008), and middle/inferior frontal gyrus (Sato et al., 2009; Tipper et al., 2008), were also involved in attentional shifts triggered by supraliminally presented eye cues. Taken together with these data, our results suggest that these 
subcortical and cortical attentional networks are involved in attentional shifts triggered by eye gaze and are more strongly activated by unconsciously than by consciously perceived eye-gaze stimuli.

Our interaction analyses also revealed that the right inferior parietal lobule was specifically involved in attentional shifts triggered by supraliminally presented eye-gaze cues. The activation of the right inferior parietal lobule is consistent with previous neuroimaging data showing that this region was active during the processing of gaze direction (e.g., Wicker et al., 1998). The greater involvement of the inferior parietal region in conscious compared with unconscious processing is also consistent with the results of a previous neuroimaging study of a brain-damaged patient, which found that this region was activated when the patient consciously perceived facial stimuli but not when he unconsciously processed these stimuli (Vuilleumier et al., 2001). Several neuroimaging (Beck et al., 2001), stimulation (Beck et al., 2006; Tseng et al., 2010), and neuroimaging + stimulation (Zaretskaya et al., 2010) studies in normal participants also found that the conscious processing of faces was associated with activation in the right parietal region. Taken together with the previous findings, our results suggest that the right inferior parietal lobule plays an important role in gaze-triggered attentional shifts involving conscious awareness.

Our results have several implications. First, the results explain behavioral data regarding gaze-triggered attentional shifts based on the neurocognitive architecture. Previous behavioral studies have shown that supraliminally and subliminally presented eyes triggered attentional shifts following a common pattern (e.g., attentional shifts are triggered by unpredictive cues under both presentation conditions; Sato et al., 2007). Our results suggest that such attentional shifts are elicited because both supraliminally and subliminally presented eyes activate the cortical attentional network. This concept is 
consistent with findings of neuroimaging studies demonstrating that the cortical attentional network is active during automatic attentional shifts irrespective of cue stimuli (e.g., Sato et al., 2009). At the same time, behavioral studies showed that supraliminally and subliminally presented eyes resulted in different patterns triggering attentional shifts, such as specific impairments of gaze-triggered attentional shifts under subliminal presentation conditions among individuals with autism spectrum disorders (Sato et al., 2010) and the opposite pattern in older participants (Baileys et al., 2014). Our results suggest that impairments of unconscious gaze-triggered attentional shifts in individuals with autism spectrum disorders may reflect reduced activity in certain brain regions, such as the amygdala, and those impairments of conscious gaze-triggered attentional shifts in older participants may reflect reduced activity in the inferior parietal lobule. These ideas are consistent with anatomical studies showing that gray matter volumes were reduced in the amygdala, but not in the inferior parietal lobule, in autistic individuals (Via et al., 2011) and age-related reductions were observed in the neocortices, including the parietal regions, but not in the amygdala (Good et al., 2001). These explanations suggest that the behavioral impairments related to gaze-triggered attentional shifts in these populations may be modified by the treatment of specific brain regions (cf. Sinha et al., 2015).

Second, our results extend the theories of neurocognitive mechanisms for attentional shifts to integrate unconscious components. The majority of traditional theories have only analyzed attentional shifts with conscious awareness (e.g., Corbetta and Shulman, 2002). Although a few researchers proposed neural mechanisms underlying both conscious and unconscious attentional shifts (e.g., Mulckhuyse and Theeuwes, 2010), they lacked evidence. Our results provide empirical support suggesting that the traditional theory of temporo-parieto-frontal cortical network for conscious attentional shifts (e.g., Corbetta and Shulman, 2002) can accommodate unconscious attentional shifts 
with the addition of some components specific to conscious and unconscious attentional shifts.

Finally, our findings have implications for the relationship between attention and consciousness. Although traditional theories have posited that attention and consciousness are tightly coupled (cf. Posner, 1994), it has also been proposed that these two components may reflect two distinct neural processes (Koch and Tsuchiya, 2007). Our data support this proposal by identifying the neural substrates of attention that are and are not accompanied by conscious awareness. Several limitations of the present study should be acknowledged. First, the differences in presentation duration between subliminally and supraliminally presented eyes may at least partially explain the present results. Although it is probably not possible to explain the commonality of the results observed across presentation conditions and those specific to the subliminal condition, the long presentation of the averted gaze may be relevant to the supraliminal-specific activation in the right inferior parietal lobule. This issue could be efficiently investigated using subliminal methods with longer stimulus presentation durations, such as the continuous flash suppression technique (Tsuchiya and Koch, 2005; Xu et al., 2011).

Second, although we found increased activity for averted versus straight eyes under both supraliminal and subliminal conditions, these results may be attributable to the task we used. Although, as mentioned above, our results under the supraliminal condition are consistent with those of several previous studies (e.g., Greene et al., 2009), other studies reported different patterns of brain activities using different tasks; for example, straight rather than averted eyes combined with emotional expressions (Sato et al., 2004) and social messages (Pelphrey et al., 2004) elicited stronger activity in the amygdala and superior temporal sulcus, respectively. To account for such discrepancies, we speculate that the relative significance of averted and straight eyes can change depending on the situation (cf. Wicker et al., 
1998) and that the eye-gaze stimuli in the cueing task we used may have increased the significance of averted eyes relative to that of straight eyes. To test such ideas, further investigations using a variety of different tasks are necessary to determine the neural mechanisms involved in the conscious and unconscious processing of eye gaze.

Finally, although the current fMRI results suggest that brain regions are rapidly activated in response to subliminally presented eyes, the exact timing of the brain activation remains unclear. Previous electrophysiological studies investigating supraliminally presented gaze have consistently reported that the temporal regions around the middle temporal gyrus show higher activity in response to averted versus straight eyes in a component peaking at around $200 \mathrm{~ms}$ (Caruana et al., 2014; Hietanen et al., 2008; McCarthy et al., 1999; Puce et al., 2000; Sato et al., 2008; Uono et al., 2014; Watanabe et al., 2001; however, see Conty et al., 2007). However, to date, no electrophysiological study has tested attentional shifts triggered by subliminally presented gaze in comparison with attentional shifts induced by supraliminally presented gaze. Future electrophysiological studies are needed to further understand the commonalities and differences in the neuro-cognitive mechanisms involved in conscious and unconscious gaze-triggered attentional shifts.

In conclusion, our conjunction analyses revealed that averted versus straight eyes activated the cortical attentional network under both supraliminal and subliminal conditions. Interaction analyses revealed that averted versus straight eyes differentially activated the cortical and subcortical regions across supraliminal and subliminal presentation conditions. These results suggest commonalities and differences in the neural mechanisms underlying conscious and unconscious attentional shifts triggered by eye gaze.

\section{Acknowledgments}


This study was supported by funds from the Benesse Corporation, the Japan Society for the Promotion of Science Funding Program for Next Generation World-Leading Researchers (LZ008), and the Organization for Promoting Research in Developmental Disorders.

\section{Conflict of interest statement}

The authors declare no competing financial or other interests.

\section{References}

Akiyama, T., Kato, M., Muramatsu, T., Saito, F., Umeda, S., Kashima, H., 2006. Gaze but not arrows: A dissociative impairment after right superior temporal gyrus damage. Neuropsychologia 44, 1804-1810.

Akiyama, T., Kato, M., Muramatsu, T., Umeda, S., Saito, F., Kashima, H., 2007. Unilateral amygdala lesions hamper attentional orienting triggered by gaze direction. Cereb. Cortex 17, 2593-2600.

Al-Janabi, S., Finkbeiner, M., 2012. Effective processing of masked eye gaze requires volitional control. Exp. Brain Res. 216, 433-443.

Ashburner, J., Friston, K.J., 2005. Unified segmentation. Neuroimage 26, $839-851$.

Bailey, P.E., Slessor, G., Rendell, P.G., Bennetts, R.J., Campbell, A., Ruffman, T., 2014. Age differences in conscious versus subconscious social perception: The influence of face age and valence on gaze following. Psychol. Aging 29 491-502.

Beck, D.M., Muggleton, N., Walsh, V., Lavie, N., 2006. Right parietal cortex plays a critical role in change blindness. Cereb. Cortex 16, 712-717.

Beck, D.M., Rees, G., Frith, C.D., Lavie, N., 2001. Neural correlates of change detection and change blindness. Nat. Neurosci. 4, 645-650.

Burra, N., Hervais-Adelman, A., Kerzel, D., Tamietto, M., de Gelder, B., Pegna, 
A.J., 2013. Amygdala activation for eye contact despite complete cortical blindness. J. Neurosci. 33, 10483-10489.

Calder, A.J., Lawrence, A.D., Keane, J., Scott, S.K., Owen, A.M., Christoffels, I., Young, A.W., 2002. Reading the mind from eye gaze. Neuropsychologia 40, $1129-1138$.

Callejas, A., Shulman, G.L., Corbetta, M., 2013. Dorsal and ventral attention systems underlie social and symbolic cueing. J. Cogn. Neurosci. 26, 63-80.

Caruana, F., Cantalupo, G., Lo Russo, G., Mai, R., Sartori, I., Avanzini, P., 2014. Human cortical activity evoked by gaze shift observation: an intracranial EEG study. Hum. Brain Mapp. 35, 1515-1528.

Cazzato, V., Macaluso, E., Crostella, F., Aglioti, S.M., 2012. Mapping reflexive shifts of attention in eye-centered and hand-centered coordinate systems. Hum. Brain Mapp. 33, 165-178.

Conty, L., N'Diaye, K., Tijus, C., George, N., 2007. When eye creates the contact! ERP evidence for early dissociation between direct and averted gaze motion processing. Neuropsychologia 45, 3024-3037.

Corbetta, M., Shulman, G.L., 2002. Control of goal-directed and stimulus-driven attention in the brain. Nat. Rev. Neurosci. 3, 201-215.

Dale, A.M., 1999. Optimal experimental design for event-related fMRI. Hum. Brain Mapp. 8, 109-114.

Duan, X., Dai, Q., Gong, Q., Chen, H., 2010. Neural mechanism of unconscious perception of surprised facial expression. Neuroimage 52, 401-407.

Ekman, P., Friesen, W.V., 1976. Pictures of Facial Affect. California: Consulting Psychologist.

Engell, A.D., Haxby, J.V., 2007. Facial expression and gaze-direction in human superior temporal sulcus. Neuropsychologia 45, 3234-3241.

Engell, A.D., Nummenmaa, L., Oosterhof, N.N., Henson, R.N., Haxby, J.V., Calder, A.J., 2010. Differential activation of frontoparietal attention 
networks by social and symbolic spatial cues. Soc. Cogn. AffectNeurosci. $5,432-440$.

Friesen, C.K., Kingstone, A., 1998. The eyes have it! Reflexive orienting is triggered by nonpredictive gaze. Psychon. Bull. Rev. 5, 490-495.

Friston, K.J., Glaser, D.E., Henson, R.N., Kiebel, S., Phillips, C., Ashburner, J., 2002. Classical and Bayesian inference in neuroimaging: Applications. Neuroimage $16,484-512$.

Friston, K.J., Holmes, A.P., Poline, J.B., Grasby, P.J., Williams, S.C., Frackowiak, R.S.J., Turner, R., 1995. Analysis of fMRI time-series revisited. Neuroimage 2, 45-53.

Friston, K.J., Josephs, O., Zarahn, E., Holmes, A.P., Rouquette, S., Poline, J., 2000. To smooth or not to smooth? Bias and efficiency in fMRI time-series analysis. Neuroimage 12, 196-208.

Friston, K.J., Zarahn, E., Josephs, O., Henson, R.N., Dale, A.M., 1999. Stochastic designs in event-related fMRI. Neuroimage 10, 607-619.

George, N., Driver, J., Dolan, R.J., 2001. Seen gaze-direction modulates fusiform activity and its coupling with other brain areas during face processing. Neuroimage 13, 1102-1112.

Good, C.D., Johnsrude, I.S., Ashburner, J., Henson, R.N., Friston, K.J., Frackowiak, R.S., 2001. A voxel-based morphometric study of ageing in 465 normal adult human brains. Neuroimage 14, 21-36.

Greene, D.J., Mooshagian, E., Kaplan, J.T., Zaidel, E., Iacoboni, M., 2009. The neural correlates of social attention: Automatic orienting to social and nonsocial cues. Psychol. Res. 73, 499-511.

Grosbras, M.H., Laird, A.R., Paus, T., 2005. Cortical regions involved in eye movements, shifts of attention, and gaze perception. Hum. Brain Mapp. 25, $140-154$

Hietanen, J.K., Leppanen, J.M., Nummenmaa, L., Astikainen, P., 2008. 
Visuospatial attention shifts by gaze and arrow cues: An ERP study. Brain Res. $1215,123-136$.

Hietanen, J.K., Nummenmaa, L., Nyman, M.J., Parkkola, R., Hamalainen, H., 2006. Automatic attention orienting by social and symbolic cues activates different neural networks: An fMRI study. Neuroimage 33, 406-413.

Hoffman, E.A., Haxby, J.V., 2000. Distinct representations of eye gaze and identity in the distributed human neural system for face perception. Nat. Neurosci. 3, 80-84.

Holmes, A.P., Friston, K.J., 1998. Generalisability, random effects and population inference. Neuroimage 7, S754.

Hooker, C.I., Paller, K.A., Gitelman, D.R., Parrish, T.B., Mesulam, M.M., Reber, P.J., 2003. Brain networks for analyzing eye gaze. Brain Res. Cogn. Brain Res. 17, 406-418.

Jiang, Y., He, S., 2006. Cortical responses to invisible faces: Ddissociating subsystems for facial-information processing. Curr. Biol. 16, 2023-2029.

Kendon, A., 1967. Some functions of gaze-direction in social interaction. Acta Psychol. 26, 22-63.

Kingstone, A., Tipper, C., Ristic, J., Ngan, E., 2004. The eyes have it!: An fMRI investigation. Brain Cogn. 55, 269-271.

Koch, C., Tsuchiya, N., 2007. Attention and consciousness: Two distinct brain processes. Trends Cogn. Sci. 11, 16-22.

Lancaster, J.L., Tordesillas-Gutiérrez, D., Martinez, M., Salinas, F., Evans, A., Zilles, K., Mazziotta, J.C., Fox, P.T., 2007. Bias between MNI and Talairach coordinates analyzed using the ICBM-152 brain template. Hum. Brain Mapp. 28, 1194-1205.

Lieberman, M.D., Cunningham, W.A., 2009. Type I and Type II error concerns in fMRI research: re-balancing the scale. Soc. Cogn. AffectNeurosci. 4, $423-428$. 
Mai, J.K., Assheuer, J., Paxinos, G., 1997. Atlas of the human brain. San Diego: Academic Press.

McCarthy, G., Puce, A., Belger, A., Allison, T. 1999. Electrophysiological studies of human face perception. II: Response properties of face-specific potentials generated in occipitotemporal cortex. Cereb. Cortex 9, 431-444. Morita, T., Itakura, S., Saito, D.N., Nakashita, S., Harada, T., Kochiyama, T., Sadato, N., 2008. The role of the right prefrontal cortex in self-evaluation of the face: A functional magnetic resonance imaging study. J. Cogn. Neurosci. 20, 342-355.

Morris, J.S., de Gelder, B., Weiskrantz, L., Dolan, R.J., 2001. Differential extrageniculostriate and amygdala responses to presentation of emotional faces in a cortically blind field. Brain 124, 1241-1252.

Morris, J.S., Öhman, A., Dolan, R.J., 1998. Conscious and unconscious emotional learning in the human amygdala. Nature 393, 467-470.

Morris, J.P., Pelphrey, K.A., McCarthy, G., 2007. Face processing without awareness in the right fusiform gyrus. Neuropsychologia 45, 3087-3091.

Mulckhuyse, M., Theeuwes, J., 2010. Unconscious attentional orienting to exogenous cues: A review of the literature. Acta Psychol. (Amst) 134, 299-309.

Okada, T., Sato, W., Kubota, Y., Usui, K., Inoue, Y., Murai, T., Hayashi, T., Toichi, M., 2008. Involvement of medial temporal structures in reflexive attentional shift by gaze. Soc. Cogn. Affect. Neurosci. 3, 80-88.

Oldfield, R.C., 1971. The assessment and analysis of handedness: The Edinburgh inventory. Neuropsychologia 9, 97-113.

Pelphrey, K.A., Singerman, J.D., Allison, T., McCarthy, G., 2003. Brain activation evoked by perception of gaze shifts: The influence of context. Neuropsychologia 41, 156-170.

Pelphrey, K. A., Viola, R. J., McCarthy, G. 2004. When strangers pass: Processing 
of mutual and averted social gaze in the superior temporal sulcus. Psychol. Sci. $15,598-603$.

Pessoa, L., Adolphs, R., 2010. Emotion processing and the amygdala: From a 'low road' to 'many roads' of evaluating biological significance. Nat. Rev. Neurosci. 11, 773-783.

Phillips, M.L., Williams, L.M., Heining, M., Herba, C.M., Russe1l, T., Andrew, C., Bullmore, E.T., Brammer, M.J., Williams, S.C., Morgan, M., Young, A.W., Gray, J.A., 2004. Differential neural responses to overt and covert presentations of facial expressions of fear and disgust. Neuroimage 21, $1484-1496$.

Posner, M.I., 1980. Orienting of attention. Q. J. Exp. Psychol. 32, 3-25.

Posner, M.I., 1994. Attention: The mechanisms of consciousness. Proc. Natl. Acad. Sci. U. S. A. 91, 7398-7403.

Price, C.J., Friston, K.J., 1997. Cognitive conjunction: A new approach to brain activation experiments. Neuroimage 5, 261-270.

Prochnow, D., Kossack, H., Brunheim, S., Muller, K., Wittsack, H.J., Markowitsch, H.J., Seitz, R.J., 2013. Processing of subliminal facial expressions of emotion: A behavioral and fMRI study. Soc. Neurosci. 8, $448-461$.

Puce, A., Allison, T., Bentin, S., Gore, J.C., McCarthy, G., 1998. Temporal cortex activation in humans viewing eye and mouth movements. J. Neurosci. 18 , $2188-2199$.

Puce, A., Smith, A., Allison, T., 2000., ERPs evoked by viewing facial movements. Cogn. Neuropsychol. 17, 221-239.

Ramasubbu, R., Konduru, N., Cortese, F., Bray, S., Gaxiola-Valdez, I., Goodyear, B., 2014. Reduced intrinsic connectivity of amygdala in adults with major depressive disorder. Front. Psychiatry 5, 17.

Rosen, A.C., Rao, S.M., Caffarra, P., Scaglioni, A., Bobholz, J.A., Woodley, S.J., 
Hammeke, T.A., Cunningham, J.M., Prieto, T.E., Binder, J.R., 1999. Neural basis of endogenous and exogenous spatial orienting. A functional MRI study. J. Cogn. Neurosci. 11, 135-152.

Sato, W., Kochiyama, T., Uono, S., Matsuda, K., Usui, K., Inoue, Y., Toichi, M., 2011. Rapid amygdala gamma oscillations in response to eye gaze. PLoS One 6, e28188.

Sato, W., Kochiyama, T., Uono, S., Matsuda, K., Usui, K., Inoue, Y., Toichi, M., 2013. Rapid and multiple-stage activation of the human amygdala for processing facial signals. Commun. Integr. Biol. 6, e24562.

Sato, W., Kochiyama, T., Uono, S., Yoshikawa, S., 2008. Time course of superior temporal sulcus activity in response to eye gaze: A combined fMRI and MEG study. Soc. Cogn. Affect. Neurosci. 3, 224-232.

Sato, W., Kochiyama, T., Uono, S., Yoshikawa, S., 2009. Commonalities in the neural mechanisms underlying automatic attentional shifts by gaze, gestures, and symbols. Neuroimage 45, 984-992.

Sato, W., Okada, T., Toichi, M., 2007. Attentional shift is triggered by gaze without awareness. Exp. Brain Res. 183, 87-94.

Sato, W., Uono, S., Okada, T., Toichi, M., 2010. Impairment of unconscious, but not conscious, gaze-triggered attention orienting in Asperger's disorder. Res. Autism Spectr. Disord. 4, 782-786.

Sato, W., Yoshikawa, S., Kochiyama, T., Matsumura, M., 2004. The amygdala processes the emotional significance of facial expressions: An fMRI investigation using the interaction between expression and face direction. Neuroimage 22, 1006-1013.

Schneider, K.A., Kastner, S., 2005. Visual responses of the human superior colliculus: A high-resolution functional magnetic resonance imaging study. J. Neurophysiol. 94, 2491-2503.

Sinha, S., McGovern, R.A., Sheth, S.A., 2015. Deep brain stimulation for severe 
autism: From pathophysiology to procedure. Neurosurg. Focus 38, E3.

Tamietto, M., de Gelder, B. 2010. Neural bases of the non-conscious perception of emotional signals. Nat. Rev. Neurosci. 11, 697-709.

Tipper, C.M., Handy, T.C., Giesbrecht, B., Kingstone, A., 2008. Brain responses to biological relevance. J. Cogn. Neurosci. 20, 879-891.

Tseng, P., Hsu, T.Y., Muggleton, N.G., Tzeng, O.J., Hung, D.L., Juan, C.H., 2010. Posterior parietal cortex mediates encoding and maintenance processes in change blindness. Neuropsychologia 48, 1063-1070.

Tsuchiya, N., Koch, C., 2005. Continuous flash suppression reduces negative afterimages. Nat. Neurosci. 8, 1096-1101.

Tzourio-Mazoyer, N. Landeau, B., Papathanassiou, D., Crivello, F., Etard, O., Delcroix, N., Mazoyer, B., Joliot, M., 2002. Automated anatomical labeling of activations in SPM using a macroscopic anatomical parcellation of the MNI MRI single-subject brain. Neuroimage 15, 273-289.

Uono, S., Sato, W., Kochiyama, T., 2014. Commonalities and differences in the spatiotemporal neural dynamics associated with automatic attentional shifts induced by gaze and arrows. Neurosci. Res. 87, 56-65.

Uono, S., Sato, W., Toichi, M., 2009. Dynamic fearful expressions enhance gaze-triggered attention orienting in high and low anxiety individuals. Soc. Behav. Pers. 37, 1313-1326.

Via, E., Radua, J., Cardoner, N., Happe, F., Mataix-Cols, D., 2011. Meta-analysis of gray matter abnormalities in autism spectrum disorder: should Asperger disorder be subsumed under a broader umbrella of autistic spectrum disorder? Arch. Gen. Psychiatry 68, 409-418.

Vuilleumier, P., Armony, J.L., Clarke, K., Husain, M., Driver, J., Dolan, R.J., 2002. Neural response to emotional faces with and without awareness: event-related fMRI in a parietal patient with visual extinction and spatial neglect. Neuropsychologia 40, 2156-2166. 
Vuilleumier, P., Pourtois, G., 2007. Distributed and interactive brain mechanisms during emotion face perception: evidence from functional neuroimaging. Neuropsychologia 45, 174-194.

Vuilleumier, P., Sagiv, N., Hazeltine, E., Poldrack, R.A., Swick, D., Rafal, R.D., Gabrieli, J.D., 2001. Neural fate of seen and unseen faces in visuospatial neglect: A combined event-related functional MRI and event-related potential study. Proc. Natl. Acad. Sci. U. S. A. 98, 3495-3500.

Watanabe, S., Kakigi, R., Puce, A., 2001. Occipitotemporal activity elicited by viewing eye movements: A magnetoencephalographic study. Neuroimage 13, $351-363$.

Whalen, P.J., Kagan, J., Cook, R.G., Davis, F.C., Kim, H., Polis, S., McLaren, D.G., Somerville, L.H., McLean, A.A., Maxwell, J.S., Johnstone, T., 2004. Human amygdala responsivity to masked fearful eye whites. Science 306, 2061.

Whalen, P.J., Rauch, S.L., Etcoff, N.L., McInerney, S.C., Lee, M.B., Jenike, M.A., 1998. Masked presentations of emotional facial expressions modulate amygdala activity without explicit knowledge. J. Neurosci. 18, $411-418$.

Wicker, B., Michel, F., Henaff, M.A., Decety, J., 1998. Brain regions involved in the perception of gaze: A PET study. Neuroimage 8, 221-227.

Xu, S., Zhang, S., Geng, H., 2011. Gaze-induced joint attention persists under high perceptual load and does not depend on awareness. Vision Res. 51, $2048-2056$.

Yang, J., Cao, Z., Xu, X., Chen, G., 2012., The amygdala is involved in affective priming effect for fearful faces. Brain Cogn. 80, 15-22.

Zaretskaya, N., Thielscher, A., Logothetis, N.K., Bartels, A., 2010. Disrupting parietal function prolongs dominance durations in binocular rivalry. Curr. Biol. 20, 2106-2111. 